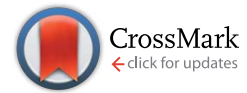

Cite this: J. Mater. Chem. A, 2015, 3, 17426

Received 12th May 2015

Accepted 21st July 2015

DOI: $10.1039 / c 5 t a 03473 f$

www.rsc.org/MaterialsA

\section{Enhanced thermoelectric performance of porous magnesium tin silicide prepared using pressure-less spark plasma sintering}

\author{
Huanpo Ning, ${ }^{a}$ Gioacchino Dario Mastrorillo, ${ }^{b}$ Salvatore Grasso, ${ }^{a}$ Baoli Du, ${ }^{a}$ \\ Takao Mori, ${ }^{c}$ Chunfeng Hu, ${ }^{c}$ Ya Xu, ${ }^{c}$ Kevin Simpson, ${ }^{d}$ Giovanni Maizza ${ }^{b}$ \\ and Michael J. Reece*a
}

Magnesium tin silicide based thermoelectrics contain earth abundant and non-toxic elements, and have the potential to replace established commercial thermoelectrics for energy conversion applications. In this work, porosity was used as a means to improve their thermoelectric properties. Compared to dense samples of $\mathrm{Sb}$ doped $\mathrm{Mg}_{2} \mathrm{Si}_{0.5} \mathrm{Sn}_{0.5}$ with a maximum $z T$ of 1.39 at $663 \mathrm{~K}$, porous samples (37\% porosity) prepared by a pressure-less spark plasma sintering technique showed significantly lower thermal conductivity and higher Seebeck coefficient, resulting in an increased maximum zT of 1.63 at $615 \mathrm{~K}$. The possible origins of the enhanced Seebeck coefficient can be attributed to a change of carrier concentration and modification of the band structure, produced by microstructural engineering of the surface composition and particle-particle contacts.

\section{Introduction}

Thermoelectric materials have attracted more and more attention worldwide because of their ability to convert energy between heat and electricity, making them suitable for applications such as power generators, and heating and cooling devices, to alleviate the increasing worldwide energy and environmental crisis. The performance of thermoelectrics is evaluated by a dimensionless figure of merit, $z T=S^{2} \sigma T / \kappa$, where $S$ is the Seebeck coefficient, $\sigma$ is the electrical conductivity, $T$ is the absolute temperature, and $\kappa$ is the thermal conductivity. A good thermoelectric material with a high $z T$ is expected to simultaneously have high Seebeck coefficient and high electrical conductivity but low thermal conductivity, as described by Slack as "electron crystal phonon glass". ${ }^{1}$ However, these three properties are interdependent and optimizing one often adversely influence the others.

Telluride based materials, such as bismuth telluride $\mathrm{Bi}_{2} \mathrm{Te}_{3}$ and lead telluride PbTe, which can have $z T$ above $1,{ }^{2-6}$ are traditional thermoelectrics widely used for near-room-temperature $(200$ to $400 \mathrm{~K})$ and mid-temperature (500 to $900 \mathrm{~K}$ ) applications, respectively. However, the growing concern regarding the toxicity and scarcity of lead and tellurium has

${ }^{a}$ School of Engineering and Materials Science, Queen Mary University of London, London, E1 4NS, UK. E-mail:m.j.reece@qmul.ac.uk

${ }^{b}$ Department of Applied Science and Technology, Institute of Materials Physics and Engineering, Corso Duca degli Abruzzi 24, 10129 Torino, Italy

${ }^{c}$ National Institute for Materials Science (NIMS), Tsukuba 305-0044\&0047, Japan

${ }^{d}$ European Thermodynamics Ltd, Leicester LE8 ORX, UK stimulated the development of high performance materials based on earth abundant and environmentally friendly elements.

Magnesium tin silicide $\left(\mathrm{Mg}_{2}(\mathrm{Si}, \mathrm{Sn})\right)$ based compounds have attracted a lot of attention as good thermoelectric candidates in recent years owing to their many advantages over traditional thermoelectrics. They contain widely abundant constituent elements ( $\mathrm{Mg}, \mathrm{Sn}, \mathrm{Si})$, with low cost and low density, and hence meet the requirements for large scale production. Their high melting point (above $1000 \mathrm{~K}$ ) enables them to be employed for high temperature applications. They generally possess $z T$ in the range of $1.0-1.3,{ }^{7-11}$ which is comparable to lead based thermoelectrics. Moreover, they are non-toxic, and environmentallyfriendly. Antimony, Sb, doping into magnesium tin silicide was reported by Liu et al. to improve its $z T$ by increasing the carrier concentration and hence electrical conductivity, while decreasing the lattice thermal conductivity. From their research, a value of $z T_{\max }=1.25$ was obtained for $\mathrm{Mg}_{2.2} \mathrm{Si}_{0.49^{-}}$ $\mathrm{Sn}_{0.5} \mathrm{Sb}_{0.01}$ at $800 \mathrm{~K} .^{7}$

Until now, most of the work to improve the thermoelectric performance of $\mathrm{Mg}_{2}(\mathrm{Si}, \mathrm{Sn})$ based materials has focused on tuning grain size, doping or substitution. ${ }^{11}$ This work aims to investigate the effect of porosity on the thermoelectric properties of $\mathrm{Sb}$ doped magnesium tin silicide $\mathrm{Mg}_{2} \mathrm{Si}_{0.5} \mathrm{Sn}_{0.5}$ (hereinafter $\left.\mathrm{Mg}_{2}(\mathrm{Si}, \mathrm{Sn})\right)$. The rationale for this approach is that porosity can lead to a significant reduction in the thermal conductivity of materials. Obviously, such an improvement might be counterbalanced by decreased electrical conductivity. Nonetheless, an increase in the Seebeck coefficient has previously been reported in the literature for certain materials with 
micro and nanoscale porosity. Du et al. reported an enhancement in the thermoelectric performance of nanoporous $\mathrm{AgSbTe}_{2}$ materials prepared by melt spinning followed by SPS. ${ }^{12}$ They found a large increase in Seebeck coefficient and attributed it to the presence of nano-sized pores, which lead to an increase in $|S|$ due to the so-called energy filtering effect. For the energy filtering effect, the pore-medium interfaces creates potential barriers, which scatters and filters low energy charge carriers, whereas the high-energy ones are almost unaffected. ${ }^{11,13}$ Therefore, $S$, which measures the average energy of electrons, is boosted by diminishing the contributions from low energy electrons. Cho et al. reported an increase in the Seebeck coefficient of $\beta-\mathrm{FeSi}_{2}$ compounds with micro-sized pores by pressure-less sintering, but a satisfactory theoretical explanation of this behavior was not given..$^{14}$ Similarly, Koumoto et al. found a large temperature dependence of the Seebeck coefficient in porous $\mathrm{SiC}$ ceramics, ${ }^{15}$ which could not be easily explained by semiconductor theory.

Spark Plasma Sintering (SPS), which uses high pulsed DC current and uniaxial pressure, has generated a lot of interest to sinter nanostructured thermoelectrics, because nanostructures could suppress the phonon contribution to thermal conductivity without a strong detrimental effect on charge transport properties. ${ }^{16-18}$ In SPS, the high heating rates minimise grain coarsening. The oxide surface layer on the particles may also be eliminated due to higher local temperature at the sintering necks. ${ }^{19}$ Porous samples can be obtained by sintering in SPS without pressure (pressure-less SPS), ${ }^{17}$ which enables fabrication of light weight and low cost components that could have better specific properties for thermoelectric generation systems. In this work, pressure-less SPS was employed to prepare porous $\mathrm{Mg}_{2}(\mathrm{Si}, \mathrm{Sn})$ samples. The thermoelectric properties of dense and porous samples were characterized and compared.

\section{Experimental procedure}

The starting powders of $\mathrm{Sb}$ doped $\mathrm{Mg}_{2} \mathrm{Si}_{0.5} \mathrm{Sn}_{0.5}$ were prepared from elemental powders ( $\mathrm{Mg}, \mathrm{Si}, \mathrm{Sn}, \mathrm{Sb})$, which were weighted, mixed, melted above their melting points in a mixer under argon and then rapidly cooled from the melt. The synthesized powder was loaded into a graphite die $(20 \mathrm{~mm}$ in diameter) and sintered in a SPS furnace (FCT, Germany) with and without pressure to produce dense and porous samples, respectively. For the dense samples, the powder was sintered at $1023 \mathrm{~K}$ under a pressure of $50 \mathrm{MPa}$ for $3 \mathrm{~min}$ at a heating rate of $100 \mathrm{~K} \mathrm{~min}^{-1}$. The temperature was controlled by a top pyrometer. For the pressure-less sintering of porous samples, the powders were poured evenly into a hollow cylindrical graphite die with internal and external diameters of 20 and $40 \mathrm{~mm}$, respectively. The hollow die containing the sample powder was inserted between two $40 \mathrm{~mm}$ diameter graphite punches and sintered in the SPS at $1123 \mathrm{~K}$ and $1173 \mathrm{~K}$ with the temperature controlled by a front pyrometer. The heating rate was $100 \mathrm{~K} \mathrm{~min}^{-1}$, and the dwell time was $2 \mathrm{~min}$. The level of vacuum during heating and cooling was around $5 \mathrm{~Pa}$.

The microstructures of the samples were observed using a scanning electron microscope (FEI, Inspect F). The X-ray diffractions (XRD) patterns were obtained with an X-ray diffractometer (Siemens, D5000) using $\mathrm{Cu} \mathrm{K} \alpha$ radiation. Samples for X-ray fluorescence (XRF) analysis were placed over a $6 \mu \mathrm{m}$ polypropylene film in sample cups and analysed on a Bruker Pioneer S4 sequential X-ray spectrometer under helium and scanned for all of the elements from Na up to U. Samples were cut into $3 \times 3 \times 12 \mathrm{~mm}^{3}$ bars for electrical resistivity and Seebeck coefficient measurements using temperature differential and four point probe methods in a partial vacuum using a measurement system (Namicro-II, China). The thermal diffusivity coefficient $(D)$ was measured on $10 \mathrm{~mm}$ diameter samples using the laser flash method (Netzsch, LFA457). A thin layer (0.1 $\mathrm{mm}$ ) of graphite paper was attached to the bottom side of the sample using highly conductive silver paste. This foil avoided the direct propagation of laser radiation through the sample. ${ }^{20}$ The room temperature carrier concentrations were determined using a Physical Properties Measurement System (PPMS, Quantum Design). The overall measurement error of the electrical and thermal transport properties was within $\pm 5 \%$.

The density of the samples was measured using the mercury infiltration method. The theoretical density of $\mathrm{Mg}_{2}(\mathrm{Si}, \mathrm{Sn})$ was calculated as $2.89 \mathrm{~g} \mathrm{~cm}^{-3}$. The specific heat was determined using differential scanning calorimetry (Netzsch, DSC 404C). The thermal conductivity was calculated from the product of thermal diffusivity, specific heat and density. The specific surface area of the samples was measured using a BET surface analyzer (Micromeritics, ASAP2020) using $\mathrm{N}_{2}$ adsorption. Prior to the measurement, the powders were heat treated at $573 \mathrm{~K}$ for 2 hours in vacuum in order to remove any moisture absorbed by the powders.

\section{Results and discussions}

Fig. 1 shows the sintering temperature versus electrical resistivity and relative density of the sintered samples measured at room temperature $\left(20^{\circ} \mathrm{C}\right)$. The samples sintered with pressure within the temperature range (823-1023 K) had relative densities exceeding $80 \%$, which increased with increasing sintering temperature. In particular, a nearly fully dense (>99\%) sample was obtained by sintering at $1023 \mathrm{~K}$. Under pressure-assisted sintering conditions, the samples sintered below $1023 \mathrm{~K}$ had high electrical resistivities exceeding $80 \mu \Omega \mathrm{m}$, so these samples were not considered for further investigation. Such high resistivities were probably due to the incomplete reduction (when sintered at low temperature) of the oxide layer on the powder particles, which acts as a passivation layer for powder storage and transport. For the pressure-less sintered porous samples, their room temperature electrical resistivity was much lower $(<20 \mu \Omega \quad \mathrm{m})$ than those of the pressure-assisted sintered samples, except for the sample pressure-assisted sintered at $1023 \mathrm{~K}(\sim 6 \mu \Omega \mathrm{m})$. This could be due to the removal of the oxide layer on the surface of the porous samples. The density of the porous samples $(62.3 \%$ and $65.7 \%$ for $\mathrm{P}-1123$ and $\mathrm{P}-1173$, respectively) also increased with sintering temperature, and their room temperature electrical resistivity was slightly higher than that of the dense sample sintered at $1023 \mathrm{~K}$. 


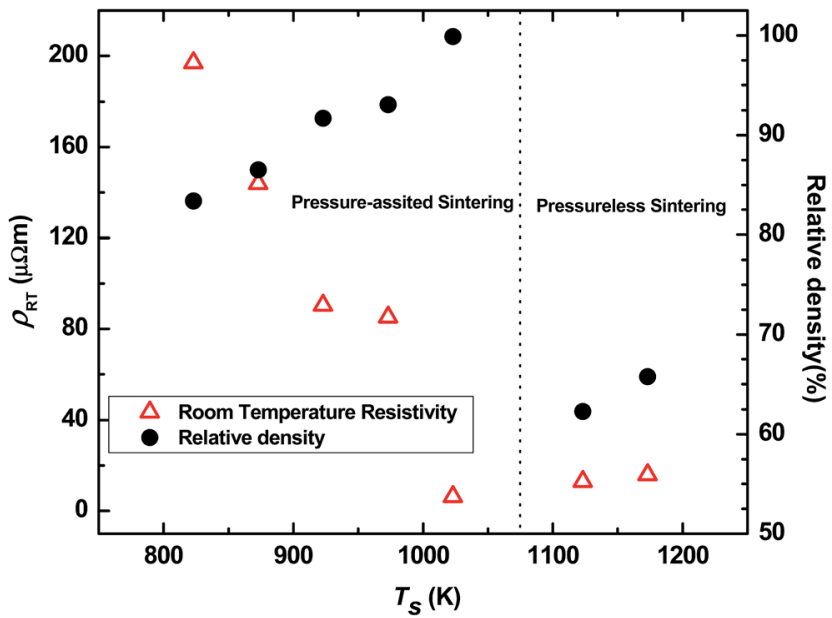

Fig. 1 Sintering temperature $\left(T_{\mathrm{s}}\right)$ versus room temperature electrical resistivity $\left(\rho_{\mathrm{RT}}\right)$ and relative density of $\mathrm{Mg}_{2}(\mathrm{Si}, \mathrm{Sn})$ samples prepared by pressure-less and pressure-assisted SPS.

The results in Fig. 1 suggest that there is a small temperature processing window that allows optimized electrical conductivity under pressure-less conditions. The plot is useful to understand the appropriate sintering conditions needed to obtain a suitable level of electrical conductivity while maximizing the degree of porosity. When using pressure assisted SPS, sintering at temperatures lower than $1023 \mathrm{~K}$ resulted in very high resistivity even if the samples had high density (93.1\%), as in the case of the sample sintered at $973 \mathrm{~K}$. In pressure-less sintering, the $\mathrm{Mg}_{2}(\mathrm{Si}, \mathrm{Sn})$ powder was heated to a temperature higher than the optimal pressure-assisted sintering temperature $(1023 \mathrm{~K})$ and held for two minutes without pressure. In this case, the densification of the sample was limited and a porous material was produced. Because the processing temperatures $(1123 \mathrm{~K}$ and $1173 \mathrm{~K})$ were close to the melting temperature, the powder started to volatize and melt, and very thin necks were formed between the particles as shown in Fig. 4. These necks could provide suitable paths for enhancing electrical conductivity. For convenience of discussion, the dense sample sintered at $1023 \mathrm{~K}$

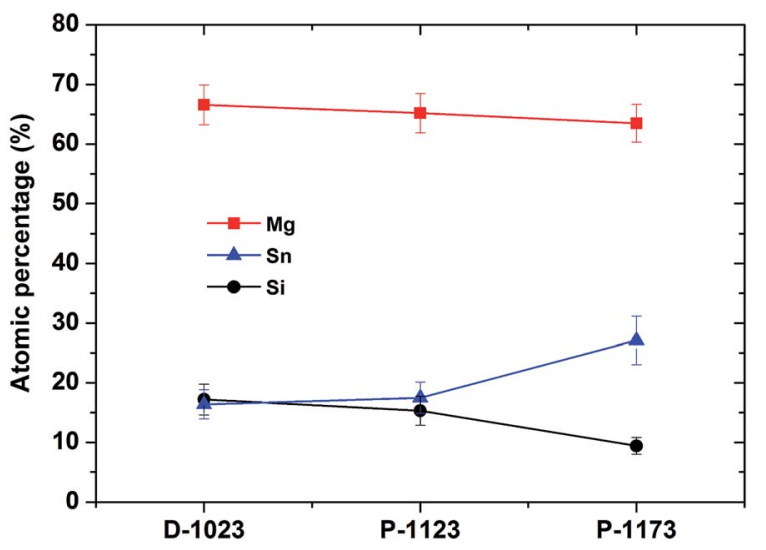

Fig. 2 XRF plot of $\mathrm{Mg}_{2}(\mathrm{Si}, \mathrm{Sb})$ dense sample $\mathrm{D}-1023$ and porous samples $\mathrm{P}-1123$ and $\mathrm{P}-1173$. and the porous samples sintered at $1123 \mathrm{~K}$ and $1173 \mathrm{~K}$ will be denoted as D-1023, P-1123, and P-1173, respectively.

The composition of the sintered samples was accurately measured using X-ray fluorescence (XRF), and the results are shown in Fig. 2. With increasing processing temperature, the atomic percentage of $\mathrm{Mg}$ and Si decreased while Sn increased. The volatilization of $\mathrm{Mg}$ is expected based on its vapour pressure versus temperature. However, the significant loss of Si at $1173 \mathrm{~K}$ is surprising and remains to be understood.

The specific surface areas (SSA) of the $\mathrm{Mg}_{2}(\mathrm{Si}, \mathrm{Sn})$ powder and porous samples were measured using a BET surface analyzer. The powder had a SSA of $2.318 \mathrm{~m}^{2} \mathrm{~g}^{-1}$, while the porous samples P-1123 and P-1173 had a SSA of 0.434 and 0.296 $\mathrm{m}^{2} \mathrm{~g}^{-1}$, respectively. The sample P-1123 had a larger surface area than P-1173, which correlates with its lower density.

Fig. 3 shows the XRD patterns of dense and porous $\mathrm{Mg}_{2}(\mathrm{Si}$, $\mathrm{Sn})$ samples after SPS sintering, with the three strongest peaks highlighted with dotted ellipses. The diffraction peaks of the dense sample D-1023 and the porous sample P-1123 can be indexed based on an available PDF card (01-089-4257) with composition $\mathrm{Mg}_{2} \mathrm{Si}_{0.4} \mathrm{Sn}_{0.6}$, although the peaks are slightly shifted due to the fact that the nominal composition of the samples was slightly different. The porous sample P-1173 showed a variation of composition, which is indicated by peak broadening and fluctuations around the main peaks, suggesting segregation of Si-rich and Sn-rich phases with compositions corresponding to between $\mathrm{Mg}_{2} \mathrm{Si}$ (PDF: 35-0773) and $\mathrm{Mg}_{2} \mathrm{Sn}$ (PDF: 65-2997).

Fig. 4 shows scanning electron microscopy (SEM) images of the as synthesized $\mathrm{Mg}_{2}(\mathrm{Si}, \mathrm{Sn})$ powder produced by rapid solidification, and also fracture surfaces of sintered samples. As shown in Fig. 4(a), the starting powders exhibited spherical morphology with a wide particle size distribution, ranging from submicrometer to tens of micrometers. The sample D-1023 had a very dense microstructure (Fig. 4(b)), while the porous samples shown in Fig. 4(c-f) have significant porosity. The main

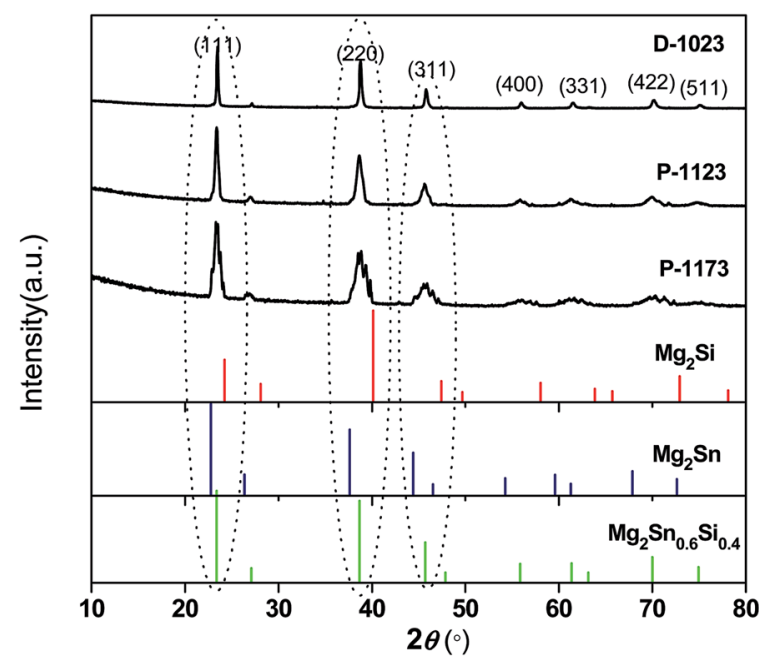

Fig. 3 XRD of dense and porous $\mathrm{Mg}_{2}(\mathrm{Si}, \mathrm{Sn})$ samples prepared by SPS compared with reference patterns. 

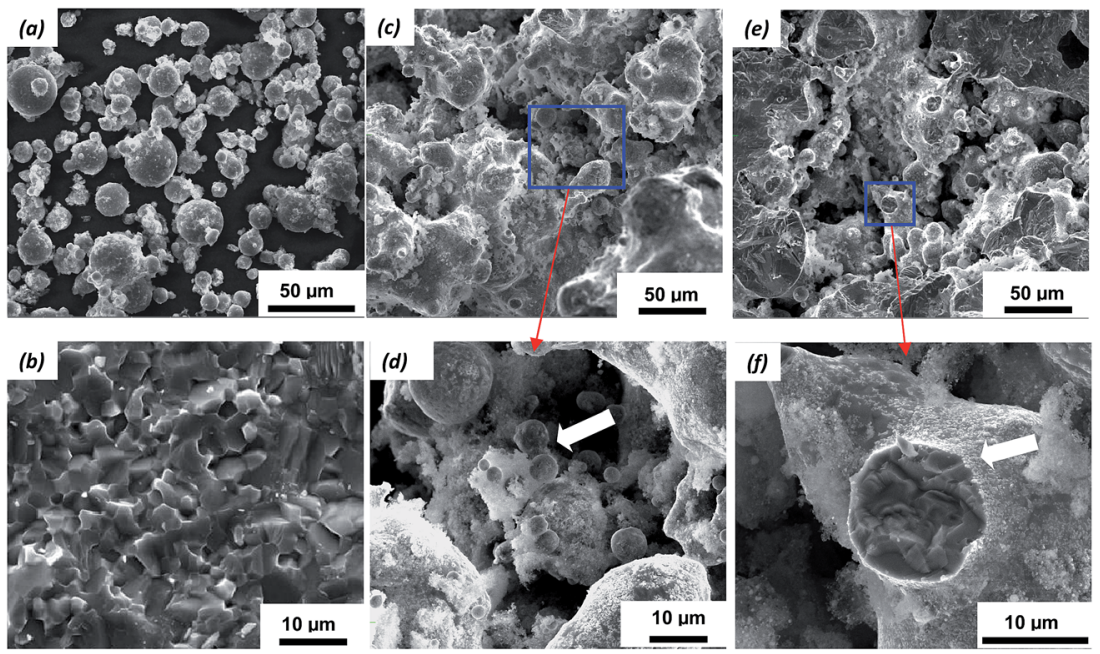

Fig. 4 SEM images of: (a) $\mathrm{Mg}_{2}(\mathrm{Si}, \mathrm{Sn})$ raw powders and (b) fractured surface of dense sample sintered at $1023 \mathrm{~K}$; (c and d) porous samples sintered at $1123 \mathrm{~K}$ and (e and f) $1173 \mathrm{~K}$ at low and high magnification.
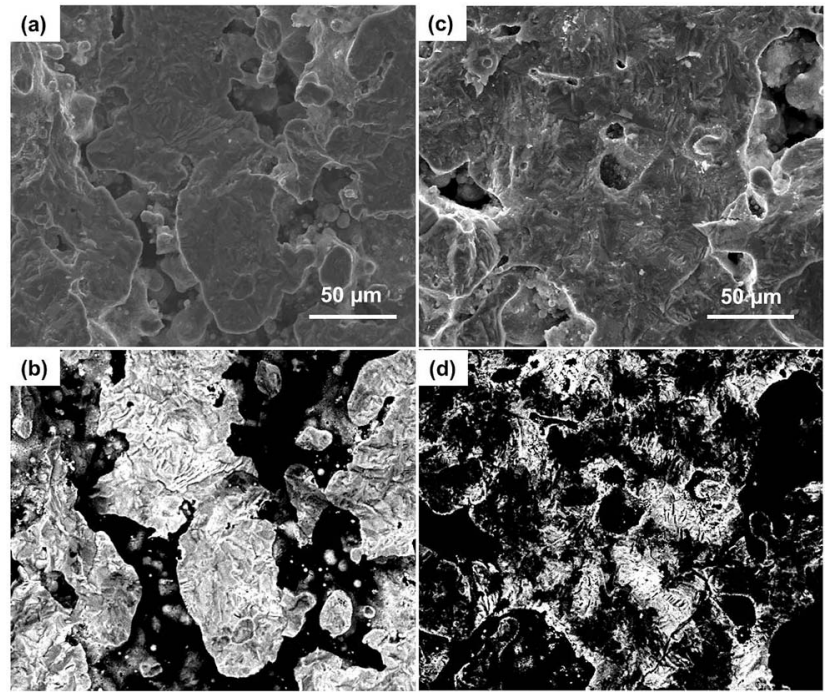

Fig. 5 (a) Secondary electron image and (b) backscattered electron image of samples P-1123; (c) secondary electron image and (d) backscattered electron image of samples P-1173.

difference between the starting powder and the porous samples is the necks formed between the sintered particles (highlighted by arrows). For the sample P-1123, the necks are barely visible and the particles seem to be weakly bonded as shown in Fig. 4(c and d), whereas for the sample P-1173 the necks are clearly visible as illustrated in Fig. 4(e and f). Fig. 5 shows the secondary and backscattered electron images of flat surfaces of the porous samples P-1123 and P-1173. It can be seen from the backscattered images that the sample $\mathrm{P}-1123$ shows almost no phase contrast, while for the sample P-1173 there is clear composition/phase segregation, which is consistent with the XRD data (Fig. 3). The bright regions of P-1173 correspond to enrichment in the highest atomic weight element, Sn, which is consistent with the XRF results (Fig. 2).
The temperature dependence of the Seebeck coefficient for the dense and porous samples is shown in Fig. 6(a). The dense sample D-1023 has similar Seebeck coefficient to those reported by Gelbstein et al. ${ }^{10}$ However, the magnitude of the Seebeck coefficient $|S|$ of the porous samples is significantly increased compared to the dense sample. The sample P-1173 exhibits a lower value of $|S|$ compared to the sample P-1123. This is probably caused by the composition variation of the sample P1173 as indicated by the XRD results (Fig. 3) and backscattered SEM image (Fig. 5(d)). The $|S|$ value for the dense sample D-1023 increases monotonically with temperature up to $762 \mathrm{~K}$, while the porous samples P-1123 and P-1173 have a maximum $|S|$ at $\sim 615 \mathrm{~K}$. The decrease of Seebeck coefficient above $615 \mathrm{~K}$ is most likely caused by the thermal excitation of minor charge carriers across the band gap at higher temperature (bipolar effect). This effect on the Seebeck coefficient is more obvious for the porous samples, which may suggest a narrowing of the effective band gap for porous samples due to impurity energy levels introduced in the band gap.

The enhanced Seebeck coefficient of the porous samples could be produced by the microstructural engineering of the surface composition and particle-particle contacts. Due to the rapid processing (dwell time $2 \mathrm{~min}$ ) during SPS processing, the loss of $\mathrm{Mg}$ is most likely to occur at the surface of the particles rather than in the bulk. The preferential compositional change occurring at the particle surface could cause a significant local change of the thermoelectric properties. Furthermore, the preferential temperature increase occurring at the particle contact points due to constriction effects can increase the Seebeck coefficient of a complex system. However, there is no clear evidence of the so-called energy filtering effect on the increase of Seebeck coefficient due to nano-sized pores. ${ }^{12}$

The relationship between Seebeck coefficient and the carrier concentration for degenerate semiconductors can be considered from the below equation, assuming the carrier mean-free path is independent of energy, ${ }^{7}$ 

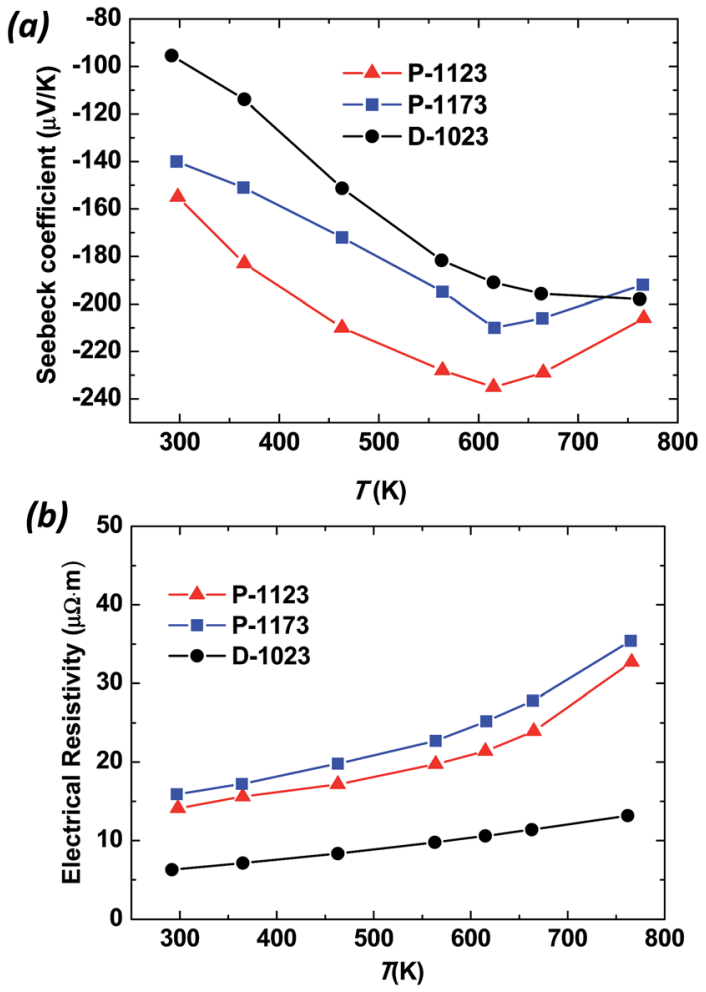

Fig. 6 Temperature dependence of: (a) Seebeck coefficient; (b) electrical resistivity of $\mathrm{Mg}_{2}(\mathrm{Si}, \mathrm{Sn})$ dense sample sintered at $1023 \mathrm{~K}$ and porous samples sintered at $1123 \mathrm{~K}$ and $1173 \mathrm{~K}$.

$$
S=\frac{8 \pi^{2} k_{\mathrm{B}}^{2}}{3 e h 2} m^{*} T\left(\frac{\pi}{3 n}\right)^{\frac{2}{3}}
$$

where $k_{\mathrm{B}}, e, h, m^{*}$ and $n$ are the Boltzmann constant, the charge of one electron, Planck's constant, the effective mass and the carrier density, respectively. It can be seen that the Seebeck coefficient is inversely proportional to the carrier concentration.

The room temperature carrier concentration data determined from the Hall effect measurements was $2.00 \times 10^{20}$ and $3.82 \times 10^{19} \mathrm{~cm}^{-3}$ for sample D-1023 and P-1073, respectively. The porous sample P-1073 has a lower electron concentration compared to the dense sample D-1023, which is consistent with the higher Seebeck coefficient of P-1073.

Another possible origin of the enhanced Seebeck coefficient is the modification of the band structure of the porous samples. Liu et al. showed that the band structure can influence the carrier density-of-states, effective mass and the Seebeck coefficient. ${ }^{8}$ The variation in the band structure could be attributed to changes in stoichiometry caused by evaporation of $\mathrm{Mg}$. It was reported by Liu et al. that in n-type $\mathrm{Mg}_{2(1+z)} \mathrm{Si}_{0.5-y} \mathrm{Sn}_{0.5} \mathrm{Sb}_{y}(0 \leq y$ $\leq 0.015$ and $0 \leq z \leq 0.15$ ) compounds, the loss of $\mathrm{Mg}$ tended to counterbalance the effect of $\mathrm{Sb}$ doping on the electrical conductivity because of the formation of $\mathrm{Mg}$ vacancies. $^{7}$ According to the thermodynamic equation: ${ }^{7}$

$$
\log P=-7550 / T-1.41 \log T+14.915 .
$$

The evaporation of $\mathrm{Mg}$ depends directly on its saturated vapour pressure $P_{\mathrm{e}}$, which increases exponentially with temperature above its melting point. For a bulk dense $\mathrm{Mg}_{2(1-\delta)} \mathrm{Si}_{1-x} \mathrm{Sb}_{x}$ sample, Tobola et al. found that increasing concentration of $\mathrm{Mg}$ vacancies $(\delta=0.1)$ could push down the Fermi level $E_{\mathrm{F}}$ from the band gap towards the valence states. ${ }^{21}$ According to the expression for the diffusive Seebeck coefficient $S,{ }^{22}$

$$
S= \pm \frac{k_{\mathrm{B}}}{e}\left[\frac{(2+\gamma) F_{1+\gamma}(\eta)}{(1+\gamma) F_{\gamma}(\eta)}-\eta\right]
$$

where $F_{\mathrm{i}}(\eta)$ stands for the Fermi integral, $\eta$ is the reduced Fermi energy $\left(=E_{\mathrm{F}} / k_{\mathrm{B}}\right), k_{\mathrm{B}}$ is the Boltzmann constant, $e$ is the charge of one electron and $\gamma$ is the scattering factor chosen to be equal to 0 for $\mathrm{Mg}_{2}$ Si-based compounds. ${ }^{7}$ The Seebeck coefficient $|S|$ will increase when the Fermi energy $E_{\mathrm{F}}$ decreases and vice versa. ${ }^{22}$ Therefore, the increased Seebeck coefficient of porous sample P-1123 and P-1173 might be partially explained by the reduction in the Fermi energy due to the deficiency of $\mathrm{Mg}$ as shown in Fig. 2 .

As shown in Fig. 6(b), the sample P-1173 had a slightly higher electrical resistivity than the sample P-1123, which is more porous. This discrepancy can also be attributed to an increased volatilization of $\mathrm{Mg}$ at a higher sintering temperature (Fig. 2), which suppressed the electron density and hence increased electrical resistivity. ${ }^{7}$ Also, as confirmed by XRD (Fig. 3), the compositional variation in the case of the sample P-1123 was minimal whereas it was apparent for the sample P-1173.

The temperature dependence of the thermal conductivity of the samples is shown in Fig. 7. The thermal conductivity was determined from measured values of thermal diffusivity and heat capacity. From Fig. 7(a), the dense sample has thermal conductivity values ( 1.5 to $2.4 \mathrm{~W} \mathrm{~m}^{-1} \mathrm{~K}^{-1}$ ) similar to those reported in the literature. ${ }^{7-11}$ The thermal conductivity curve for the porous samples P-1123 and P-1173 are quite similar and the values are dramatically reduced compared to those of the dense sample. The minimum value of $1.0 \mathrm{~W} \mathrm{~m}^{-1} \mathrm{~K}^{-1}$ at $615 \mathrm{~K}$ for the porous samples is much lower than the dense sample with a minimum value of $1.5 \mathrm{~W} \mathrm{~m}^{-1} \mathrm{~K}^{-1}$ at the same temperature. The upturn of the total thermal conductivity at around $615 \mathrm{~K}$ is probably due to the increased bipolar contribution as the concentration of minority holes increases when the temperature approaches the intrinsic conduction region. ${ }^{9}$ To better understand all the mechanisms contributing to the total thermal conductivity, the total thermal conductivity $\kappa_{\mathrm{t}}$ can be divided as $\kappa_{\mathrm{t}}=\kappa_{\mathrm{L}}+\kappa_{\mathrm{e}}+\kappa_{\mathrm{b}}$, where $\kappa_{\mathrm{L}}, \kappa_{\mathrm{e}}$ and $\kappa_{\mathrm{b}}$ are the lattice, electronic and bipolar contributions to the total thermal conductivity, respectively. ${ }^{9}$ Based on the Wiedemann-Franz law, the electronic thermal conductivity, $\kappa_{\mathrm{e}}$, can be estimated from the equation $\kappa_{\mathrm{e}}=L_{0} \sigma T$, where $L_{0}, \sigma$ and $T$ are the Lorenz number $\left(L_{0}=1.7\right)$, the electrical conductivity and the absolute temperature, respectively. The inset in Fig. 7(a) shows the electronic contribution $\kappa_{\mathrm{e}}$ to the total thermal conductivity. It can be seen that the electronic contribution is relatively stable for all the samples and gradually increases with increasing temperature until around $670 \mathrm{~K}$ and then decreases slowly. The $\kappa_{\mathrm{e}}$ is higher for the dense sample D-1023 due to its higher electrical conductivity. For the porous samples, the electronic 
(a)

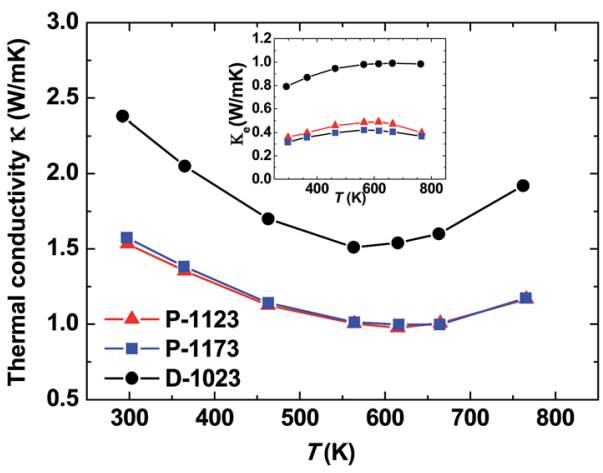

(b)

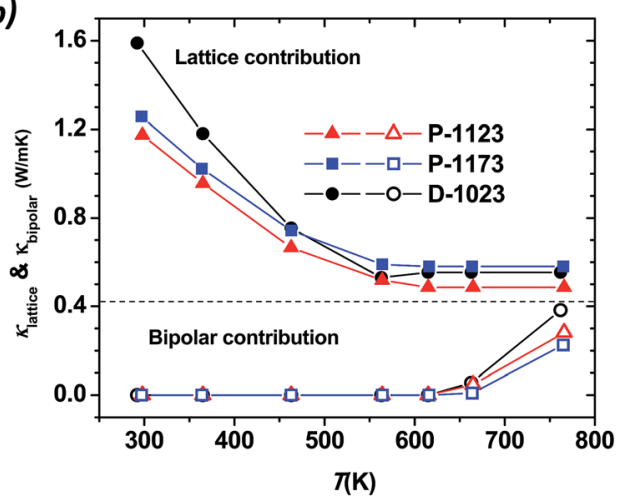

Fig. 7 Temperature dependence of: (a) total thermal conductivity $\kappa_{\mathrm{t}}$ with inset electronic contribution $\kappa_{\mathrm{e}}$ to the total thermal conductivity; (b) lattice contribution $\kappa_{\mathrm{L}}$ and bipolar contribution $\kappa_{\mathrm{b}}$ to total thermal conductivity of $\mathrm{Mg}_{2}(\mathrm{Si}, \mathrm{Sn})$ dense sample sintered at $1023 \mathrm{~K}$, and porous samples sintered at $1123 \mathrm{~K}$ and $1173 \mathrm{~K}$.

contribution to the thermal conductivity is suppressed by a factor of 2, which is proportional to its increased electrical resistivity compared to the dense material.

The lattice contribution $\kappa_{\mathrm{L}}$ generally decreases with increasing temperature due to increased phonon scattering, and eventually saturates at a certain temperature. The bipolar contribution $\kappa_{\mathrm{b}}$ is typically very small below the intrinsic conduction region of

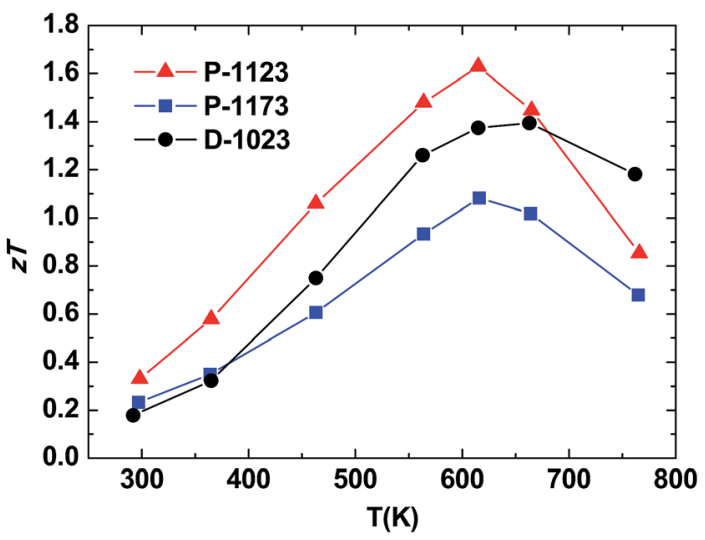

Fig. 8 Temperature dependence of figure of merit $z T$ for $\mathrm{Mg}_{2}(\mathrm{Si}, \mathrm{Sn})$ dense sample sintered at $1023 \mathrm{~K}$, and porous samples sintered at 1123 $\mathrm{K}$ and $1173 \mathrm{~K}$. materials. ${ }^{23}$ By assuming that the lattice contribution $\kappa_{\mathrm{L}}$ becomes constant above $615 \mathrm{~K}$ and that the bipolar contribution $\kappa_{\mathrm{b}}$ can be neglected below $615 \mathrm{~K},{ }^{18}$ the two contributions can be estimated as shown in Fig. 7(b). The lattice contribution $\kappa_{\mathrm{L}}$ is higher for the dense sample D-1023 than for the porous samples at below $473 \mathrm{~K}$. This is because the pores of the porous material induced stronger phonon scattering. Above $573 \mathrm{~K}$, the sample P-1123 has the lowest lattice thermal conductivity $\kappa_{\mathrm{L}}\left(\sim 0.486 \mathrm{~W} \mathrm{~m}^{-1} \mathrm{~K}^{-1}\right)$. The dense sample has a higher bipolar contribution $\kappa_{\mathrm{b}}$ than the porous samples. Compared to its influence on Seebeck coefficient (Fig. 6(a)), the bipolar effect on the thermal conductivity of porous samples is less significant. The reason for this is not clear, but it may be related to the porosity effects.

The temperature dependence of $z T$ is shown in Fig. 8. As the temperature increases, $z T$ increases and reaches a maximum. For the porous samples P-1123 and P-1173, the maximum $z T$ is 1.63 and 1.08 at $\sim 615 \mathrm{~K}$, respectively. The dense sample D-1023 shows a maximum $z T$ of about 1.39 at a higher temperature ( $663 \mathrm{~K})$. The maximum $z T$ of the porous material P-1123 is higher than the dense sample with a composition $\mathrm{Mg}_{2.2} \mathrm{Si}_{0.49^{-}}$ $\mathrm{Sn}_{0.5} \mathrm{Sb}_{0.01}$ and $z T_{\max }=1.25$ reported by Liu et al. ${ }^{7}$

\section{Conclusions}

Porous samples of $\mathrm{Sb}$ doped $\mathrm{Mg}_{2} \mathrm{Si}_{0.5} \mathrm{Sn}_{0.5}$ were prepared using a pressure-less spark plasma sintering technique. The porous samples (porosity $<37 \%$ ) showed significantly reduced thermal conductivity and enhanced Seebeck coefficient compared to dense samples. The fundamental mechanisms of the enhanced Seebeck coefficient are not clear, but the possible origins can be attributed to the change of carrier concentration and modification in the band structure, produced by microstructural engineering of the surface composition and particle-particle contacts. As a result of reduced thermal conductivity and enhanced Seebeck coefficient, the porous samples showed a higher $z T$ than a dense sample up to $670 \mathrm{~K}$, with a maximum $z T$ $=1.63$ at $\sim 615 \mathrm{~K}$ for a porous sample sintered at $1123 \mathrm{~K}$ with a relative density of $63 \%$.

\section{References}

1 G. A. Slack, CRC Handbook of Thermoelectrics, ed. D. M. Rowe, CRC Press, Boca Raton, FL, 1st edn, 1995.

2 Z. H. Dughaish, Phys. B, 2002, 322, 205-223.

3 J. P. Heremans, V. Jovovic, E. S. Toberer, A. Saramat, K. Kurosaki, A. Charoenphakdee, S. Snyder and G. J. Yamanaka, Science, 2008, 321, 554-557.

4 B. Poudel, Q. Hao, Y. Ma, Y. C. Lan, A. Minnich, B. Yu, X. A. Yan, D. Z. Wang, A. Muto, D. Vashaee, X. Y. Chen, J. M. Liu, M. S. Dresselhaus, G. Chen and Z. F. Ren, Science, 2008, 320, 634-638.

5 S. Grasso, N. Tsujii, Q. Jiang, J. Khaliq, S. Maruyama, M. Miranda, K. Simpson, T. Mori and M. J. Reece, J. Mater. Chem., 2013, 1, 2362-2367.

6 Q. Jiang, H. Yan, J. Khaliq, H. Ning, S. Grasso, K. Simpson and M. J. Reece, J. Mater. Chem. A, 2014, 2, 5785-5790. 
7 W. Liu, X. Tang, H. Li, J. Sharp, X. Zhou and C. Uher, Chem. Mater., 2011, 23, 5256-5263.

8 W. Liu, X. Tan, K. Yin, H. Liu, X. Tang, J. Shi, Q. Zhang and C. Uher, Phys. Rev. Lett., 2012, 108, 166601.

9 W. Liu, X. Tang, H. Li, K. Yin, J. Sharp, X. Zhou and C. Uher, J. Mater. Chem., 2012, 22, 13653.

10 Y. Gelbstein, J. Tunbridge, R. Dixon, M. J. Reece, H. Ning, R. Gilchrist, R. Summers, I. Agote, M. A. Lagos, K. Simpson, C. Rouaud, P. Feulner, S. Rivera, R. Torrecillas, M. Husband, J. Crossley and I. Robinson, J. Electron. Mater., 2014, 43, 1703-1711.

11 M. B. A. Bashira, S. M. Saidb, M. F. M. Sabria, D. A. Shnawaha and M. H. Elsheikha, Renewable Sustainable Energy Rev., 2014, 37, 569-584.

12 B. Du, H. Li, J. Xu, X. Tang and C. Uher, J. Solid State Chem., 2011, 184, 109-114.

13 H. Lee, D. Vashaee, D. Z. Wang, M. S. Dresselhaus, Z. F. Ren and G. Chen, J. Appl. Phys., 2010, 107, 094308.

14 W. Cho and K. Park, J. Mater. Sci.: Mater. Electron., 2000, 11, 319-324.
15 K. Koumoto and M. Shimoigoshi, J. Mater. Sci. Lett., 1987, 6, 1453-1455.

16 E. Savary, F. Gascoin, S. Marinel and R. Heuguet, Powder Technol., 2012, 228, 295-300.

17 S. Grasso, Y. Sakka and G. Maizza, Sci. Technol. Adv. Mater., 2009, 10, 053001.

18 J. G. Noudem, S. Lemonnier, M. Prevel, E. S. Reddy, E. Guilmeau and C. Goupil, J. Eur. Ceram. Soc., 2008, 28, 41-48.

19 O. Guillon, J. Gonzalez-Julian, B. Dargatz, T. Kessel, G. Schierning, J. Rathel and M. Herrmann, Adv. Eng. Mater., 2014, 16, 830-849.

20 Z. Zivcova, E. Gregorova, W. Pabst, D. S. Smith, A. Michot and C. Poulier, J. Eur. Ceram. Soc., 2009, 29, 347-353.

21 J. Tobola, H. Schrrer and S. Kaprzyk, J. Electron. Mater., 2010, 39, 2064-2069.

22 P. Pichanusakorn and P. Bandaru, Mater. Sci. Eng., R, 2010, 67, 19-63.

23 B. Du, Y. Saiga, K. Kajisa and T. Takabatake, J. Appl. Phys., 2012, 111, 013707. 